\title{
Erratum zu: Biosphäre 4.0
}

\author{
Axel Borsdorf, Michael Jungmeier, Valerie Braun und Kati Heinrich
}

\section{Erratum zu:}

\section{A. Borsdorf et al. (Hrsg.), Biosphäre 4.0, https://doi.org/10.1007/978-3-662-60707-7}

Affiliation of Florian Knaus in chapter 6 Akzeptanz, Identifikation und Engagement: ... chapter 18 Welchen Beitrag kann ein Biosphere Reserve zur Umsetzung leisten? ... Institut für Terrestrische Ökosysteme, ETH Zürich, Zürich, Schweiz und Biosphärenmanagement, UNESCO Biosphäre Entlebuch (UBE), Schüpfheim, Schweiz

Affiliation of Annette Schmid in chapter 18 Welchen Beitrag kann ein Biosphere Reserve zur Umsetzung leisten? ... Biosphärenmanagement, UNESCO Biosphäre Entlebuch (UBE), Schüpfheim, Schweiz

Die korrigierten Versionen der Kapitel sind verfügbar unter https://doi.org/10.1007/978-3-662-60707-7_6 https://doi.org/10.1007/978-3-662-60707-7_18 